\title{
Diseño de una mesa elevadora para el traslado de material maquinado, que permita evitar fatiga en el operador
}

\section{Design of a lifting table for the transfer of machined material, which allows to avoid fatigue in the operator}

\author{
FLORES-GALVÁN, Francisco Ángel*†, NAVA-MORALES, Francisca, ALVA-GALLEGOS, Rodrigo \\ y BALTAZAR-PLATA, Carlos Gustavo
}

Universidad Tecnológica del Valle de Toluca, Lerma, México.

ID $1^{\text {er }}$ Autor: Francisco Ángel, Flores-Galván / ORC ID: 0000-0001-9434-4610

ID $1{ }^{\text {er }}$ Coautor: Francisca, Nava-Morales / ORC ID: 0000-0001-8931-6770, CVU CONACYT ID: 507130

ID $2^{\text {do }}$ Coautor: Rodrigo, Alva-Gallegos / ORC ID: 0000-0002-9354-2006, CVU CONACYT ID: 333455

ID $3{ }^{\text {er }}$ Coautor: Carlos Gustavo, Baltazar-Plata / ORC ID: 0000-0003-4131-7138

DOI: $10.35429 / J I D .2019 .8 .3 .17 .23$

Recibido 09 Julio, 2019; Aceptado 03 de Septiembre, 2019

\begin{abstract}
Resumen
Objetivo: Diseñar una plataforma de elevación hidráulica mediante el uso del software SOLIDWORKS, para agilizar el traslado de cajas de material maquinado y evitar daños físicos. Metodología: 1. Análisis del problema que tienen los trabajadores al trasladar material maquinado a sus respectivas áreas de trabajo, haciendo hincapié en la mala manipulación que realizan. 2. Descripción de los diferentes tipos de plataformas elevadoras móviles de personal (PEMP) que existen en el mercado. 3. Realizar un muestreo para precisar el peso promedio que adquieren los materiales después de ser maquinados y justificar que son cargas elevadas y difíciles de manipular sin que cause un daño físico. 4. Estudio de fuerzas en SolidWorks, con el propósito de observar si, es un mecanismo resistente y si la carga que de desea suministrar no generara pandeos en las vigas. 5. Diseño y moldeo de una plataforma elevadora móvil de carga, y su validación a través del programa de elementos finitos SOLIDWORKS, que mejorará la ergonomía en la distribución del material maquinado a su próximo destino, evitando lesiones irreversibles y agilizando dicha actividad. 6. Evaluación de los costos que se generan al fabricar la mesa elevadora y los beneficios económicos que tendrá. Contribución: Evitar daños físicos ocasionados por el mal manejo de cargas y que finalmente genera problemas de producción.
\end{abstract}

Carga Daño, Diseño

\begin{abstract}
Objective: Design a hydraulic lifting platform through the use of SOLIDWORKS software, to expedite the transfer of boxes of machined material and avoid physical damage. Methodology 1. Analysis of the problem that workers have when moving machined material to their respective work areas, emphasizing the bad manipulation they perform. 2 . Description of the different types of mobile personnel lifting platforms (PEMP) that exist in the market. 3 . Perform a sampling to specify the average weight that the materials acquire after being machined and justify that they are high loads and difficult to handle without causing physical damage. 4. Study of forces in SolidWorks, with the purpose of observing if, it is a resistant mechanism and if the load you wish to supply will not generate buckling on the beams. 5. Design and molding of a mobile lifting platform, and its validation through the SOLIDWORKS finite element program, which will improve the ergonomics in the distribution of the machined material to its next destination, avoiding irreversible injuries and speeding up said activity. 6. Evaluation of the costs generated by manufacturing the lift table and the economic benefits it will have. Contribution: Avoid physical damage caused by the mishandling of loads and that ultimately generates production problems.
\end{abstract}

Load, Damage, Design

Citación: FLORES-GALVÁN, Francisco Ángel, NAVA-MORALES, Francisca, ALVA-GALLEGOS, Rodrigo y BALTAZAR-PLATA, Carlos Gustavo. Diseño de una mesa elevadora para el traslado de material maquinado, que permita evitar fatiga en el operador. Revista del Diseño Innovativo. 2019. 3-8: 17-23

\footnotetext{
* Correspondencia del Autor (Correo electrónico: francisco.flores@utvtol.edu.mx)

$\dagger$ Investigador contribuyendo como primer autor.
} 


\section{Analisis del problema}

En el área de producción se genera un sinfín de materiales maquinados (Figura 1) de diversas formas, tamaños, y diverso material con diferentes pesos y geometrías, que son almacenados en cajas (Figura 2) para después de concluida la jornada de producción sean trasladadas a la siguiente estación de trabajo, o a inspección final.

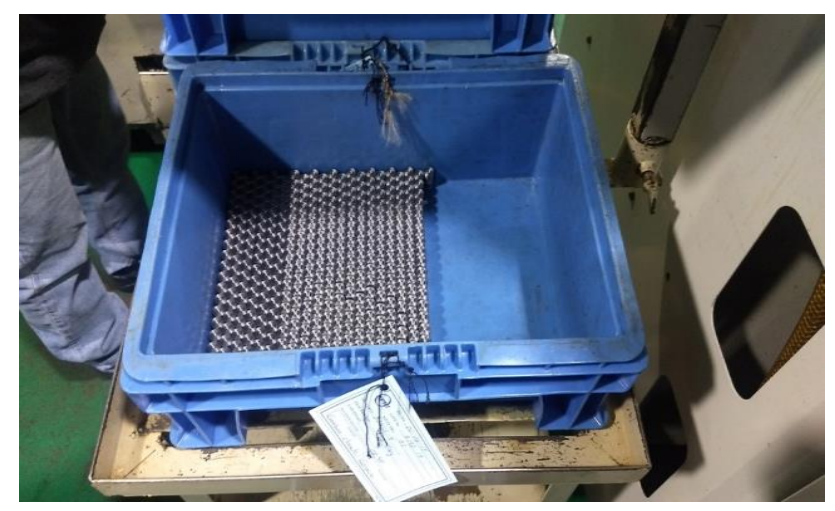

Figura 1 Material maquinado

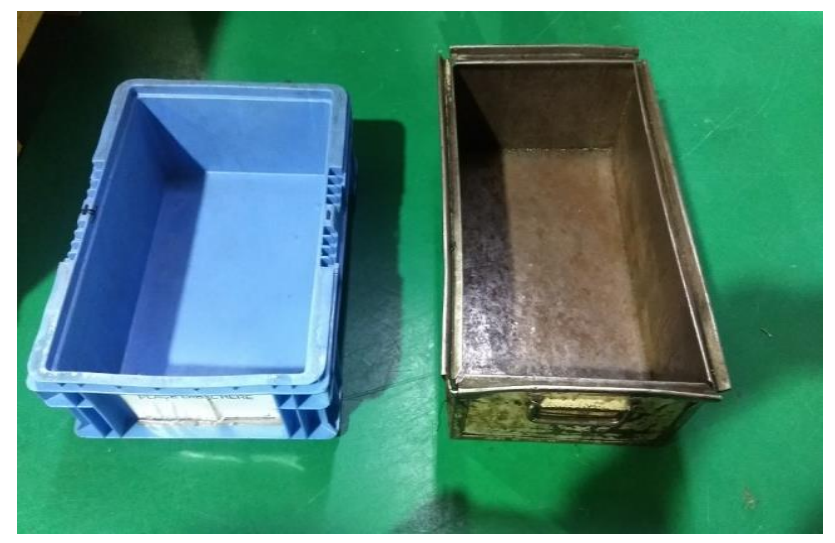

Figura 2 Tipos de cajas

Cada operario tiene la tarea, al finalizar la jornada, de trasladar su material al área delimitada con cinta (Figura 3) que este más cercana a su lugar de trabajo, para después ser recogido por quienes llevarán dicho material maquinado a su siguiente operación o a inspección final. Pero esta tarea resulta ser perjudicial para quien la realiza pues de ser llevada a cabo de la manera incorrecta puede ocasionar en el personal problemas en la columna que resultan irreversibles.

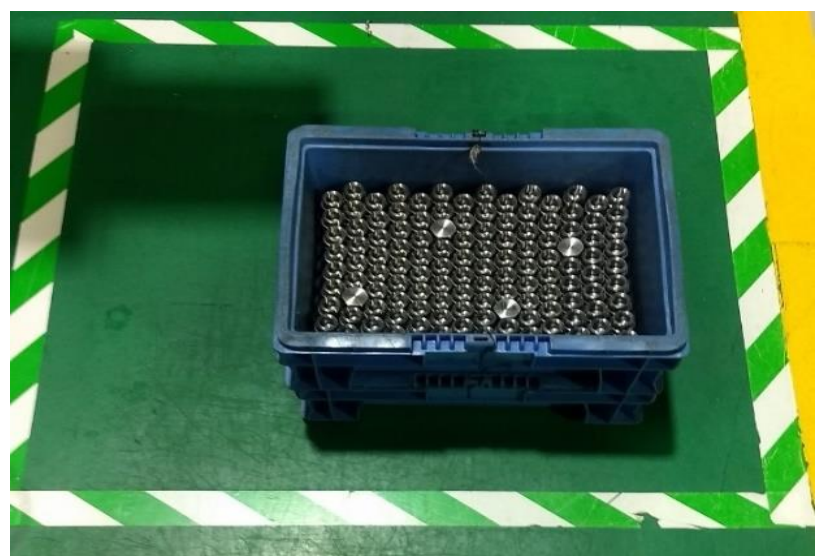

Figura 3 Área delimitada

Quienes tienen la labor de trasladar el material a su siguiente destino no tienen la técnica para levantar cargas de manera adecuada y evitar lesiones (Figura 4), además de no contar con el equipo de protección para llevar a cabo dicha tarea, como lo son las fajas sacrolumbares y/o cinturones. Se ha observado que los trabajadores que cumplen la tarea de abastecer de material a cada operación no se preocupan de usar este equipo, se desconoce si la empresa no lo ha proporcionado o si a pesar de contar con el equipo adecuado simplemente evitan portarlo.

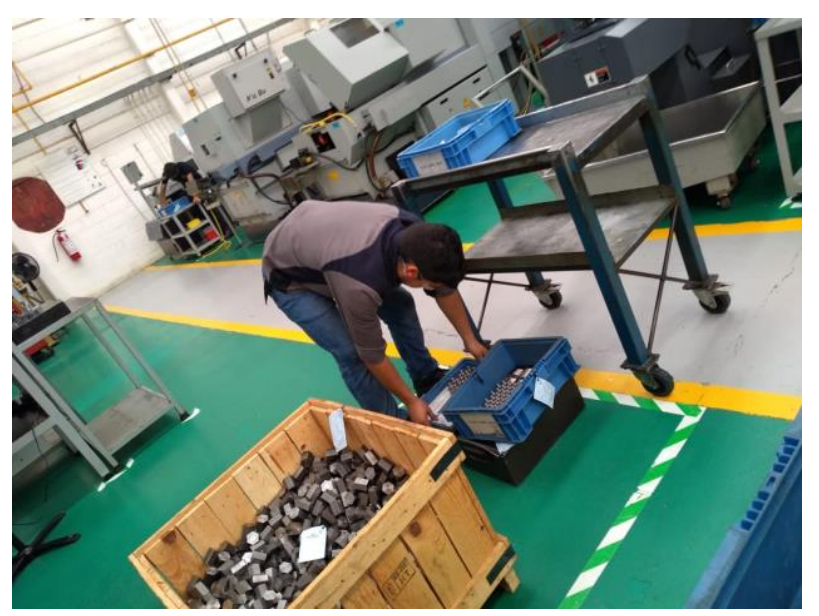

Figura 4 Manera errónea como se manipula la carga

\section{Diferentes tipos de plataformas elevadoras móviles}

Se han desarrollado mecanismos para lograr conseguir dicho fin de la forma más cómoda y segura posible. Gracias al desarrollo a su vez de la hidráulica y los sistemas a presión desde hace ya mucho tiempo se dispone de la fuerza necesaria para levantar pesos elevados facilitando enormemente muchas tareas que requerían lidiar con ellos. 
Dentro del mundo de las plataformas elevadoras móviles (PEM), existen muchos tipos distintos, tanto en morfología como en capacidad y desempeño a la hora de ser utilizadas en labores o situaciones concretas (Figura 5) Según la norma UNE-EN 280, los elevadores destinados a uso de personal (PEMP) se clasifican en función de la proyección vertical del centro de gravedad (c.d.g) de la carga en:

Grupo A: aquellas cuya proyección vertical del centro de gravedad de la carga con la que se esté trabajando se encuentra siempre dentro de las líneas de vuelco, teniendo en cuenta todas las posibles configuraciones de la máquina y a la máxima inclinación del chasis especificada por el fabricante.

Grupo B: aquellas PEMP que no son grupo A. Se definen líneas de vuelco como aquellas que delimitan la región en la que se encuentra la base o apoyo del sistema elevador. Existe según la norma una segunda clasificación complementaria a la primera en función del tipo de traslación que permiten las PEMP:

Tipo 1: La traslación solo se puede realizar si la PEMP se encuentra en posición de transporte.

Tipo 2: La traslación de la PEMP cuando la plataforma se encuentra en posición elevada solo es posible realizarla desde un dispositivo situado en el chasis.

Tipo 3: La traslación de la PEMP cuando la plataforma se encuentra en posición elevada es posible realizarla desde un dispositivo situado en la plataforma.

Cabe mencionar que los tipos 2 y 3 pueden aparecer presentes en una misma máquina.

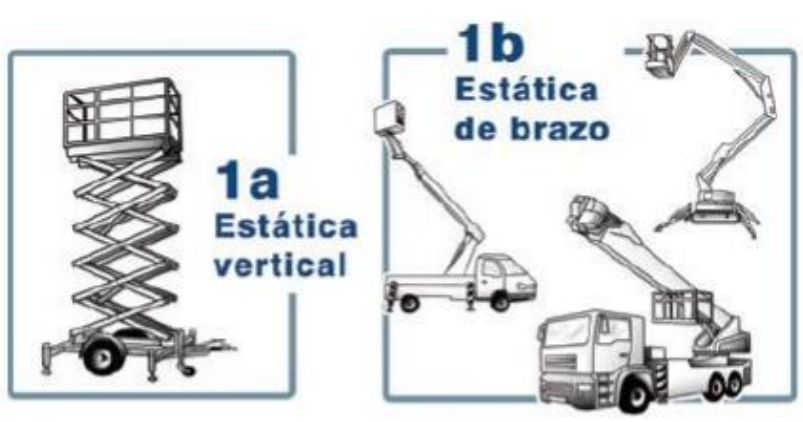

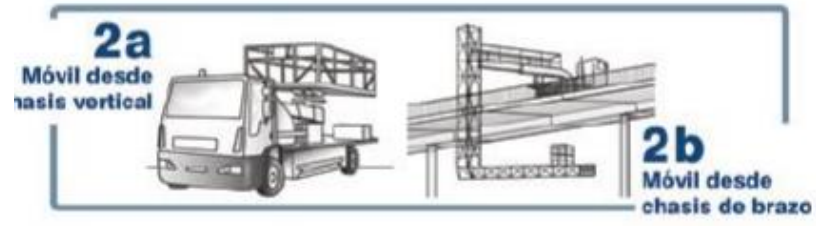
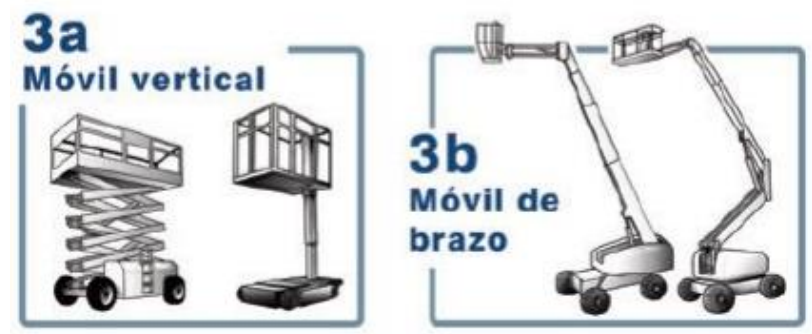

Figura 5 Tipos de PEMP

Se ha escogido una grúa de tipo Tijera ya que supone una gran ayuda para trabajos de elevación de cargas pesadas y de grandes dimensiones. Algunas de las características que reúnen este tipo de máquinas son las siguientes: Este tipo de elevadores son de posición fija ya que para su desplazamiento se precisa de otra máquina que lo eleve y lo desplace. Se puede aumentar o disminuir la altura de trabajo con gran facilidad. Se trata de una máquina de gran rigidez, esto da al operario una mayor sensación de seguridad que resulta en un trabajo más eficaz. A la hora de alcanzar la altura de trabajo, en esta plataforma sólo es necesario accionar el sistema hidráulico para elevar la estructura de tijera.

\section{Muestreo del peso de los materiales maquinados}

Para obtener un promedio del peso que adquieren las cajas con diferentes tipos de material maquinado, se realizó un muestreo con cinco diferentes piezas. Se pesaron los materiales antes de ser maquinados y después de realizada la correspondiente operación obteniendo así el peso total que tendrá la caja después de terminada la jornada.

El peso máximo que un trabajador puede manipular manualmente es 25 kilos. (Tabla 1 ). 


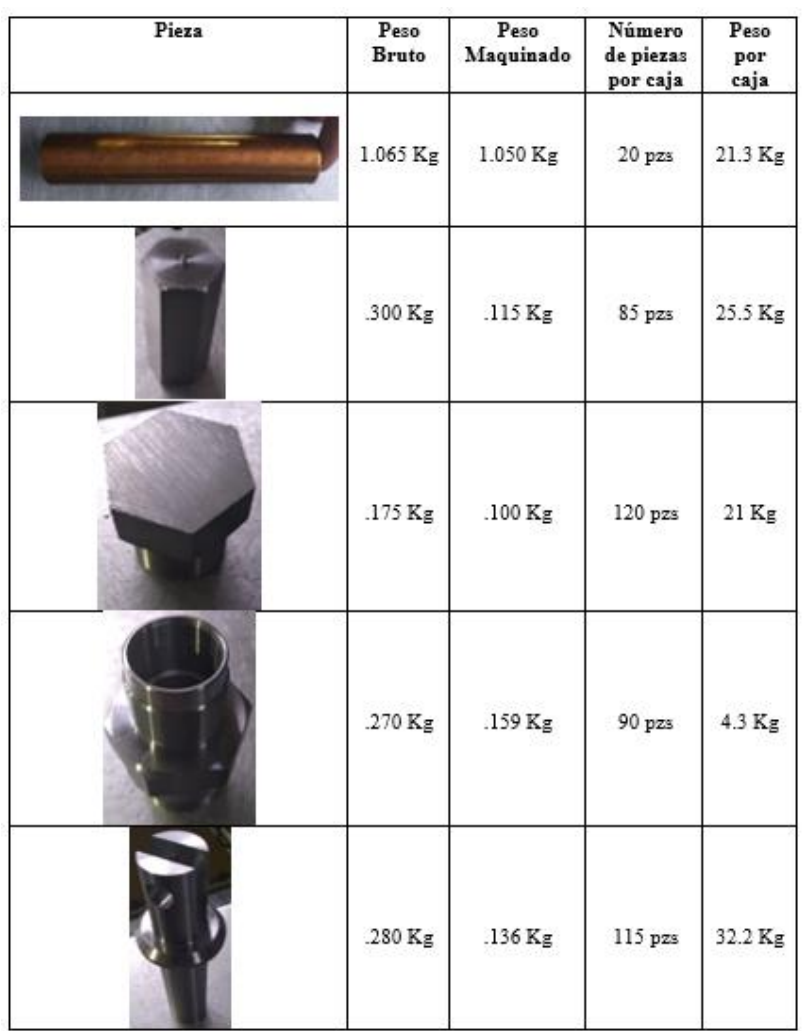

Tabla 1 Muestreo del peso por caja de material maquinado

\section{Estudio de fuerzas en SolidWorks}

Al tratarse de una plataforma elevadora fija, el diseño de esta máquina debe cumplir con la normativa UNE-EN 12158-1:2001+A1. Esta norma se titula "Elevadores de obras de construcción para cargas. Parte 1: elevadores con plataformas accesibles". Para el cálculo y diseño de esta plataforma se han utilizado estas normas como guía para definir las soluciones propuestas.

En estas se indican los parámetros de diseño con los que se llevan a cabo, los cálculos para determinar los esfuerzos a los que está sometida la estructura. De esta forma se podrá realizar el dimensionamiento de cada componente asegurando que soportan las solicitaciones y garantizando la estabilidad de la máquina en las distintas condiciones de trabajo sin que se produzca riesgo de vuelco.

Los parámetros de diseño dependen de las dimensiones principales de la grúa que son: el largo, el alto y el ancho. Por ellos primero fijamos éstos y a partir de ellos y con las condiciones establecidas en las normas determinamos las fuerzas que actúan en nuestra grúa tijera.
Factores de diseño:
- Carga nominal: se trata de los $300 \mathrm{~kg}$ que tendrá que soportar el elevador.
Cargas debidas al viento: las despreciamos.
Velocidad de elevación: $1.5 \mathrm{~m}$ en 10 segundos.

Cuando se tengan los mayores esfuerzos en cada elemento se podrá dimensionar cada uno de ellos ya que una vez calculada la geometría necesaria para la peor situación de carga, esta geometría será válida para el resto de las situaciones de esfuerzos.

La grúa tiene dos estructuras tijera a cada lado de la plataforma, es simétrica, por lo tanto, cada una de las estructuras sobre que se apoya absorberá la mitad de la carga. Lo que se calcula a continuación, es la carga que absorbe una de las tijeras (Figura 6):

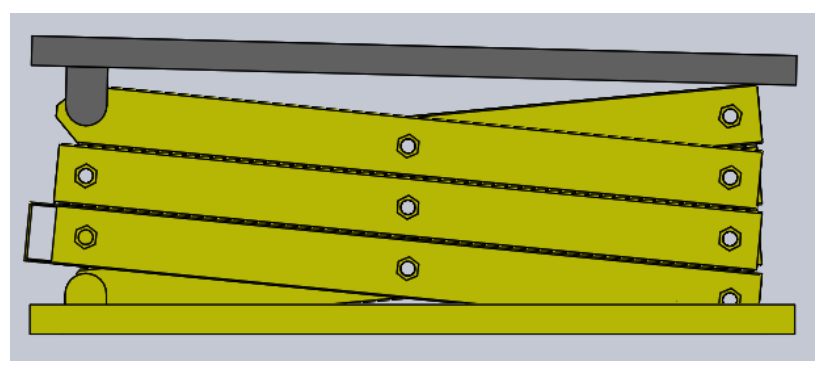

Figura 6 Primer caso de carga, posición inferior

Se emplean estos apoyos dado que el apoyo $\mathrm{B}$ tiene bloqueado el desplazamiento en las direcciones $\mathrm{X}, \mathrm{Y}, \mathrm{Z}$, pero si permite el giro en Z. En cambio, el apoyo A solo permite el giro en $\mathrm{Z}$ y el desplazamiento en $\mathrm{X}$. (Figura 7) de acuerdo a Oberg Erik, D. Franklin \& Holbrook L [1].

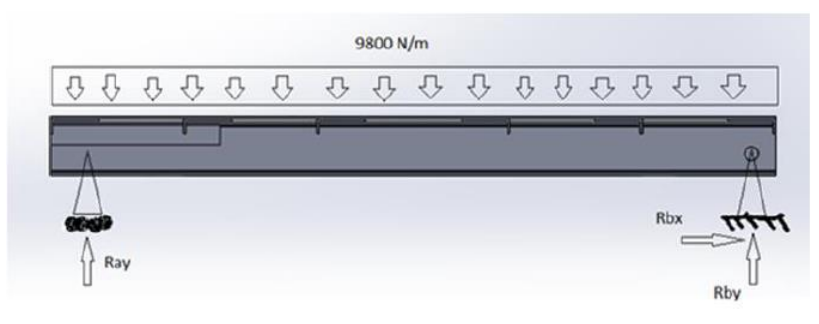

Figura 7 Esfuerzos en los apoyos superiores

Se calculan los esfuerzos en los apoyos superiores de la mesa: 
Ecuaciones de equilibrio de la Figura 28:

$\Sigma F x=0 R b x=0$

$\Sigma F y=0$ Ray + Rby $-9800 * 3=0$

$\Sigma M b=0-R a y * 3+(9800 * 3 * 1,5)=0$

Se obtiene que Ray $=$ Rby $=14700$ N.

Para una solo tijera $\mathrm{Ray}=\mathrm{Rby}=7350 \mathrm{~N}$

Se observa que el ángulo de las barras con la horizontal cuando está cerrada $\alpha=6,18$ grados.

\section{Resultados del diseño}

El primer requisito del diseño fue determinar la cantidad de tijeras que se iban a emplear para alcanzar la altura de diseño, 1.5 metros. (Figura 7)

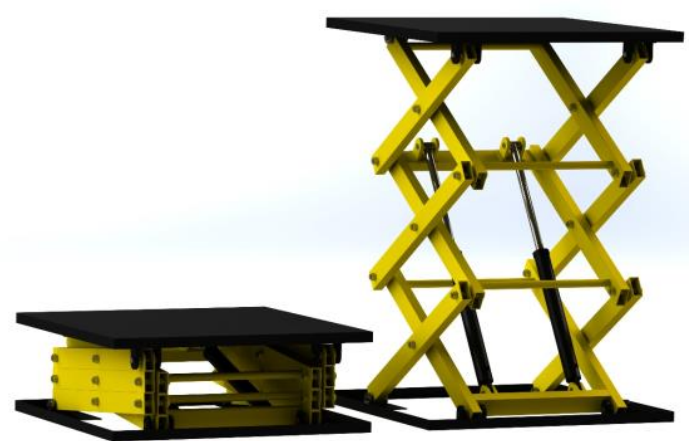

Figura 7 Visualización de posiciones

Como primera opción y dado que las dimensiones perimetrales de la base de la tijera eran de [50 x 40 centímetros] se propuso una sola tijera. Esta solución no fue posible dado que esta tendría que encontrarse totalmente verticales para alcanzar la altura deseada. Esto llevo a que se optara por colocar tres tijeras, las cuales alcanzaran un ángulo de $29^{\circ}$, como máximo lo que llevaría en su conjunto a que la altura máxima de la base superior alcanzara los 1.5 metros. (Figura 8 y Figura 9)

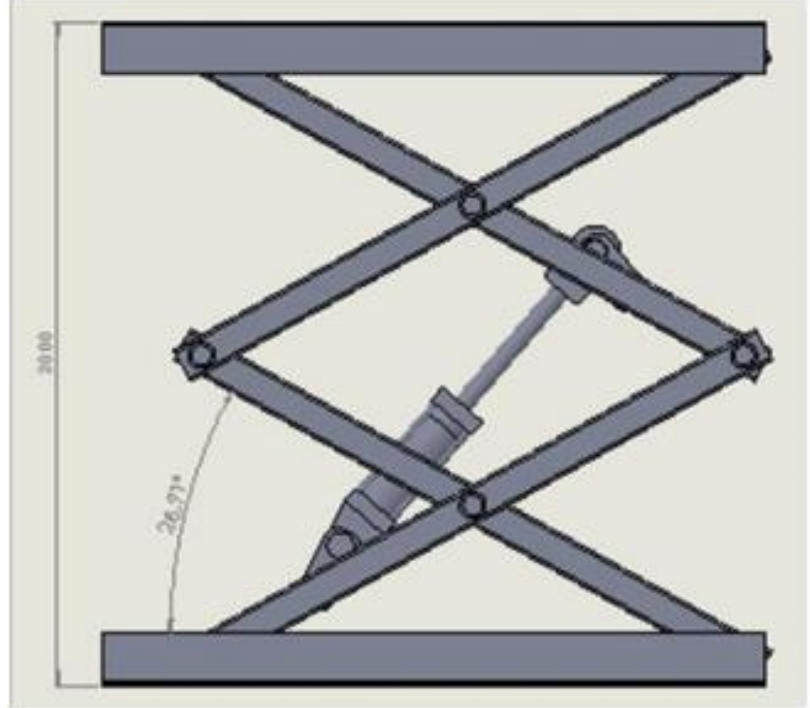

Figura 8 Lateral del elevador tijera

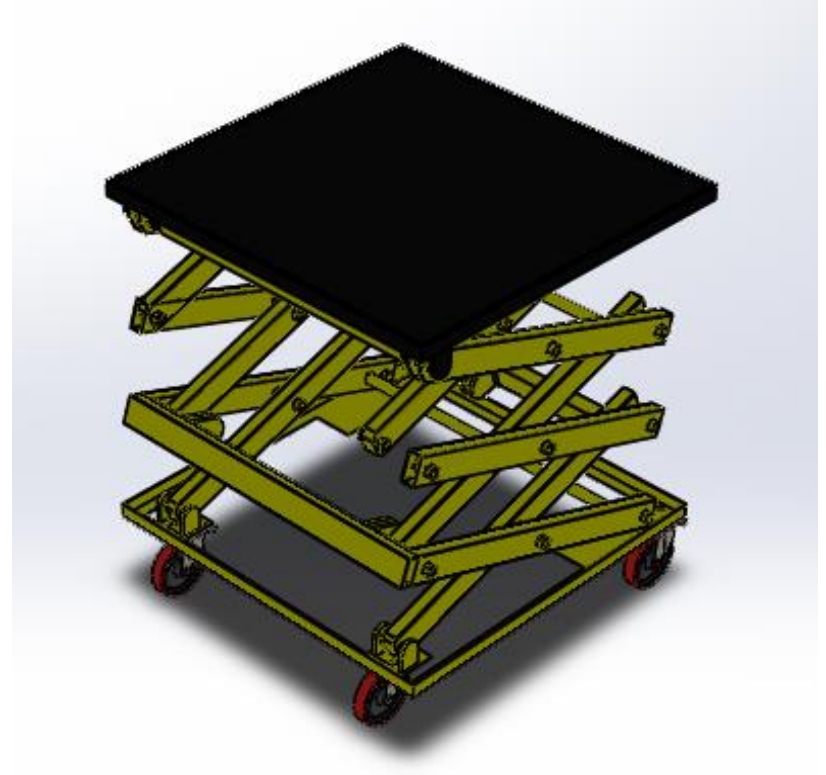

Figura 9 Diseño con las ruedas colocadas

\section{Comprobación de resistencia a la fuerza ideal de carga}

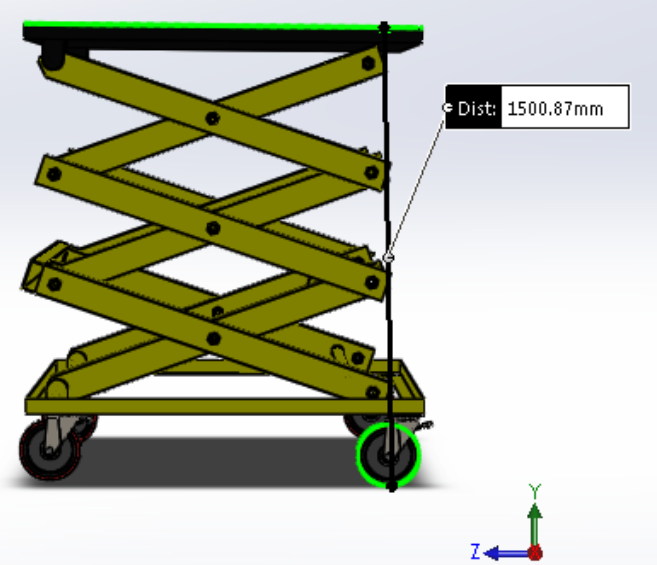

FLORES-GALVÁN, Francisco Ángel, NAVA-MORALES, Francisca, ALVA-GALLEGOS, Rodrigo y BALTAZAR-PLATA, Carlos Gustavo. Diseño de una mesa elevadora para el traslado de material maquinado, que permita evitar fatiga en el operador. Revista del Diseño Innovativo. 2019 
La altura de la plataforma completa se eleva a $1.500 .87 \mathrm{~mm}$ que convertido a metros seria 1.5 metros. Por consiguiente, para poder dar una estimación de los esfuerzos sometidos tomamos una fórmula para poder convertir el peso a Newtons fuerza y esta es: (Figura 10)

\section{$\mathbf{W}=\mathbf{m} \times \mathbf{g}$}

$\mathrm{W}=$ Peso, se da en newtons

$\mathrm{M}=$ Masa, se da en $\mathrm{Kg}$.

$\mathrm{G}=$ Gravedad, se toma como $9.8 \mathrm{~m}$ sobre segundo a cuadrado $\left(\mathrm{m} / \mathrm{s}^{\wedge} 2\right)$

$\mathrm{W}=300 \mathrm{Kg} \times 9.8 \mathrm{~m} / \mathrm{s}^{\wedge} 2$

$\mathrm{W}=2940 \mathrm{~N}$.

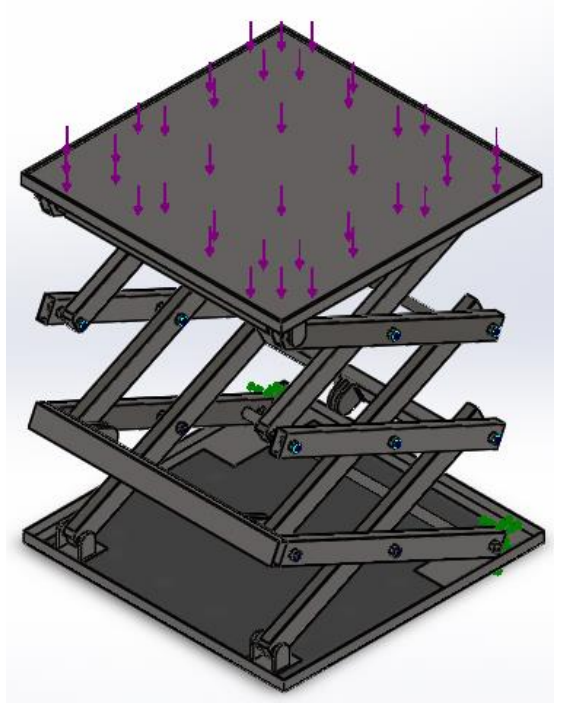

Figura 10 Fuerzas ejercidas

Se tomaran para la fuerza a la que se someterá la plataforma se toma en cuenta la malla para poder definir las fuerzas por partes y visualizar las fallas a las que se somete la plataforma.(Figura 11)

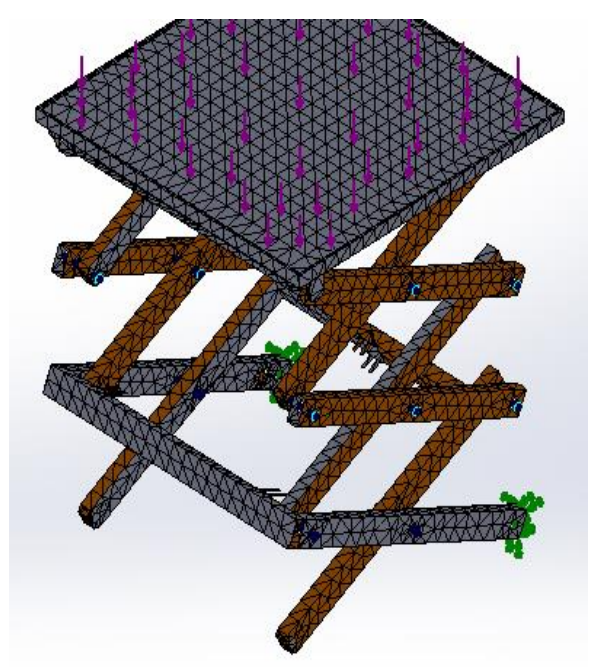

Figura 11 Resistencia de la plataforma

\section{De acuerdo a [2] BALLAST TM.}

Se debe conocer la fuerza de empuje que se quiere ejercer en el circuito hidráulico. Que será de: 300 kilos.

Calcularemos la capacidad de empuje de un cilindro de 69.2 (Vástago) X70 (Interior del tubo) X68 (Carrera).

300 kilos $=661.38$ libras

Radio $=7.6 \mathrm{~cm}=2.99$ in

Área efectiva $=3.14(1.49 * 1.49)=6.97$ in2

Presión $=661.38$ libras $/ 6.97 \mathrm{plg} 2=94.88 \mathrm{psi}$

$94.88 \mathrm{psi}=6.54 \mathrm{bar}$

Adquirir ruedas de caucho estándar giratoria con freno, con las siguientes características:

Rueda de Caucho Estándar - Giratoria con Freno, 4 x 1 1/4"

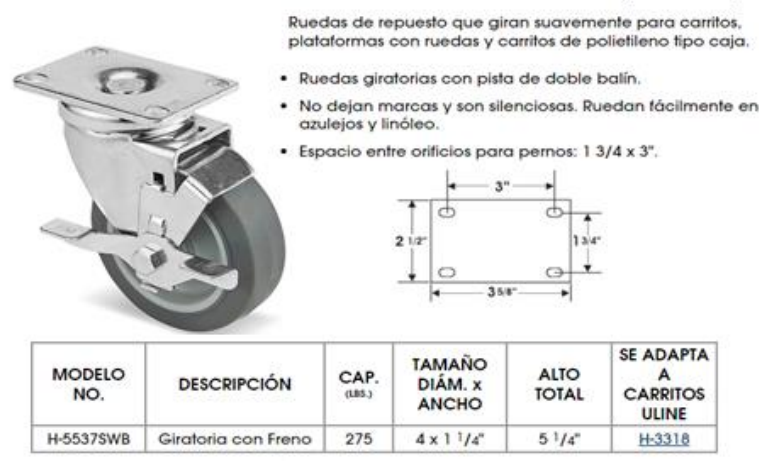

\section{Estudio de factibilidad}

\begin{tabular}{|l|l|l|l|}
\hline \multicolumn{1}{|c|}{ Material } & Cantid ad & $\begin{array}{c}\text { Costo } \\
\text { por pieza }\end{array}$ & $\begin{array}{l}\text { Costo } \\
\text { final }\end{array}$ \\
\hline Lamina de 5 mm & 1 pieza & 120 & 89 \\
\hline $\begin{array}{l}\text { Tubo de ace ro } \\
\text { rectangular }\end{array}$ & 4 piezas & 127 & 507 \\
\hline Tornillos \%/ & 32 piezas & 1.28 & 42 \\
\hline Perno \% $\%$ & 20 piezas & 8 & 160 \\
\hline $\begin{array}{l}\text { Rueda giratoria con } \\
\text { cerradura y 8"de } \\
\text { diámetro piezas }\end{array}$ & 260 & 1040 \\
\hline Tubo de acero solido & 2 piezas & 450 & 450 \\
\hline Cilindro hidráulico & 2 piezas & 2,500 & 5,000 \\
\hline Gran Total & & & 7288 \\
\hline
\end{tabular}

Tabla 3 Lista de materiales y su costo

En la fabricación en planta del carro de tijera se invierte: 
Mano de obra $\$ 200$ x día

Ensamble hecho en 3 días como máximo $\$ 600+7,228=\$ 7,828$ pesos

\begin{tabular}{|l|l|l|}
\hline \multicolumn{1}{|c|}{ Designación } & Material & Norma \\
\hline $\begin{array}{l}\text { Anillo de retención } \\
\text { interno }\end{array}$ & & DIN 472 \\
\hline $\begin{array}{l}\text { Anillo de retención } \\
\text { externo }\end{array}$ & & DIN 471 \\
\hline $\begin{array}{l}\text { Eje de rueda } \\
\text { superior }\end{array}$ & AISI 1045 & \\
\hline $\begin{array}{l}\text { Placa base sujeción de } \\
\text { mesa de para }\end{array}$ & AISI 1020 & AISI 1020 \\
\hline $\begin{array}{l}\text { Barra perfil PHR } \\
160.120 .7\end{array}$ & AISI 1045 \\
\hline $\begin{array}{l}\text { Casquillo } \\
\text { pasadores }\end{array}$ & & AISI 1035 \\
\hline
\end{tabular}

Tabla 4 Lista de materiales y normas

El análisis costo-beneficio refleja que una inversión será rentable cuando la relación costo-beneficio sea mayor que la unidad (ya que los beneficios serán mayores que los costos de inversión), y no será rentable cuando la relación costo-beneficio sea igual o menor que la unidad (ya que los beneficios serán iguales o menores que los costos de inversión): La fórmula de la relación costo-beneficio es:

\section{$\mathrm{B} / \mathrm{C}=\mathrm{VAI} / \mathrm{VAC}$}

En donde:

B/C: relación costo-beneficio.

VAI: valor actual de los ingresos totales netos o beneficios netos.

VAC: valor actual de los costos de inversión o costos totales.

Considerando que el posible daño físico incapacite al operador dos meses en un periodo de un año, el costo sería de $\$ 12,000$ sabiendo que el operador percibe un salario de 200 pesos diarios.

$\mathrm{B} / \mathrm{C}=12000 / 7828=1.53$

Por lo que la manufactura del carrito es totalmente viable.

\section{Conclusiones}

Considerando el impacto que el carro de transporte hace en el manejo seguro del producto terminado donde los operadores harán un traslado fácil evitando fatiga y posibles daños a la columna vertebral además de un acomodo eficaz del material en la mesa ya que las medidas de la misma fueron hechas de acuerdo a las dimensiones de las cajas de almacenamiento, el proyecto es viable de construir.

\section{Referencias}

[1] Oberg Erik, D. Franklin \& Holbrook L. (2010). Machinery's Handbook. New York,N.Y.: INDUSTRIAL PRESS INC.

[2] BALLAST TM. Rescatado de: http://spanish.telescopic-

hydrauliccylinder.com/sale-10534635-trunnioneye-pin-multi-stage-single-acting-telescopichydraulic-cylinder.html 